PHYSICAL REVIEW FLUIDS 2, 090511 (2017)

\title{
Flight of a falling maple seed
}

\author{
Injae Lee and Haecheon Choi ${ }^{*}$ \\ Department of Mechanical and Aerospace Engineering, Seoul National University, Seoul 08826, Korea \\ (Received 11 August 2017; published 29 September 2017)
}

\begin{abstract}
This paper is associated with a video winner of a 2016 APS/DFD Gallery of Fluid Motion Award. The original video is available from the Gallery of Fluid Motion, https://doi.org/10.1103/APS.DFD.2016.GFM.V0046
\end{abstract}

DOI: 10.1103/PhysRevFluids.2.090511

In autumn, maple trees disperse their seeds by employing autorotation under windy conditions. Maple seeds equipped with a small wing begin to autorotate within $1 \mathrm{~m}$ after they are detached from trees, and the autorotating motion generates the lift force that slows down its descent $[1,2]$. From measurements using digital particle image velocimetry (DPIV), it was elucidated that a stable leading-edge vortex (LEV) is generated by the rotating wing, allowing it to attain a high lift force [1]. However, due to complex vortical structures behind maple seeds, their dynamic behaviors have not been fully understood.

To understand the falling mechanism together with the flow characteristics, we conduct an unsteady three-dimensional numerical simulation of a freely falling maple seed. For the simulation, a three-dimensional seed model [Fig. 1(a)] is obtained by scanning a maple seed (Acer palmatum), and we assume that the density of the maple seed is uniform over the nut and wing areas. We use an immersed boundary method in a noninertial reference frame [3] fixed to the center of mass of the seed. Numerical details are given in [4]. Figure 1(b) shows the time traces of descending and rotating velocities of the maple seed during free fall. Initially, the descending velocity increases almost linearly under the gravity, and then decreases as the rotating velocity develops (autorotation). Finally, the seed reaches a periodic state with terminal descending and rotating velocities.

Figure 2(a) shows the trajectories of the center of mass (CM) and wing tip (WT) of the freely falling seed model. After a transient period, the seed falls with the periodic rotating motion. Figures $2(\mathrm{~b})$ and 2(c) show the instantaneous three-dimensional vortical structures around the seed at two different time instants. Before periodic autorotation, vortices are shed from the base edge of the seed and the wake zone is narrow [Fig. 2(b)]. In the periodic state, due to the rotating motion of the wing, the leading-edge (LEV), wing-tip (WTV), wing-root (WRV), and trailing-edge (TEV) vortices are generated, and the wake zone is broad [Fig. 2(c)]. In particular, the LEV remains stable along the span, rather than sheds into the wake. This creates a high lift force, resulting in a significant reduction of the descent velocity [1]. Therefore, all the vortices except the LEV dominate the wake behind the seed.

\footnotetext{
*Corresponding author: choi@ snu.ac.kr
}

Published by the American Physical Society under the terms of the Creative Commons Attribution 4.0 International license. Further distribution of this work must maintain attribution to the author(s) and the published article's title, journal citation, and DOI. 


\section{INJAE LEE AND HAECHEON CHOI}

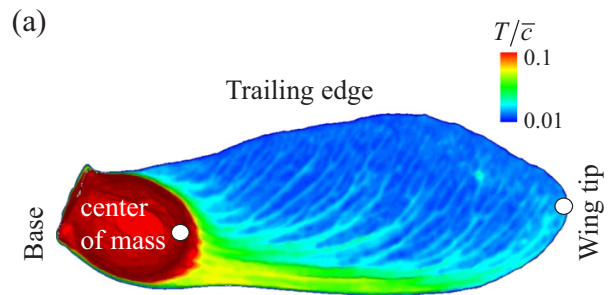

Leading edge (b)

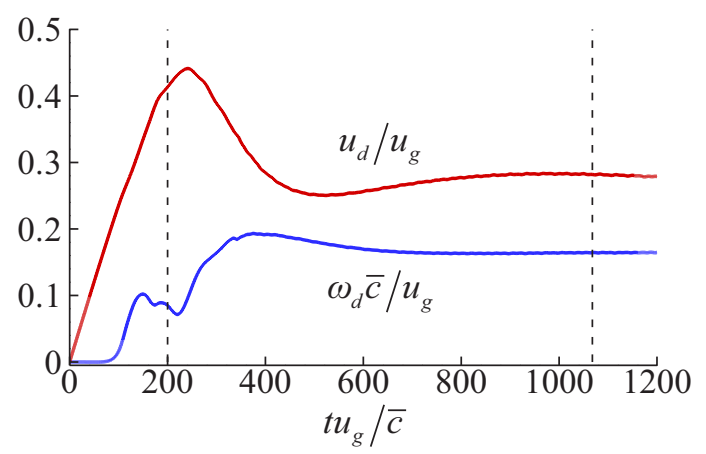

FIG. 1. (a) Seed model constructed by three-dimensional scanning; (b) time traces of descending $\left(u_{d} / u_{g}\right)$ and rotating $\left(\omega_{d} \bar{c} / u_{g}\right)$ velocities of the maple seed during free fall. Here, $u_{g}=\sqrt{(\rho-1) \bar{c} g}$ (characteristic terminal velocity), $\rho=\rho_{s} / \rho_{f}=404.7$ (ratio of solid to fluid densities), $\bar{c}=A_{w} / s=4.272 \mathrm{~mm}$ (mean chord length), $g$ is the gravitational acceleration, and $A_{w}$ and $s$ are the wing area and span of the seed, respectively. Colors in (a) denote the thickness distribution of the seed. DOI: https://doi.org/10.1103/APS.DFD.2016.GFM.V0046

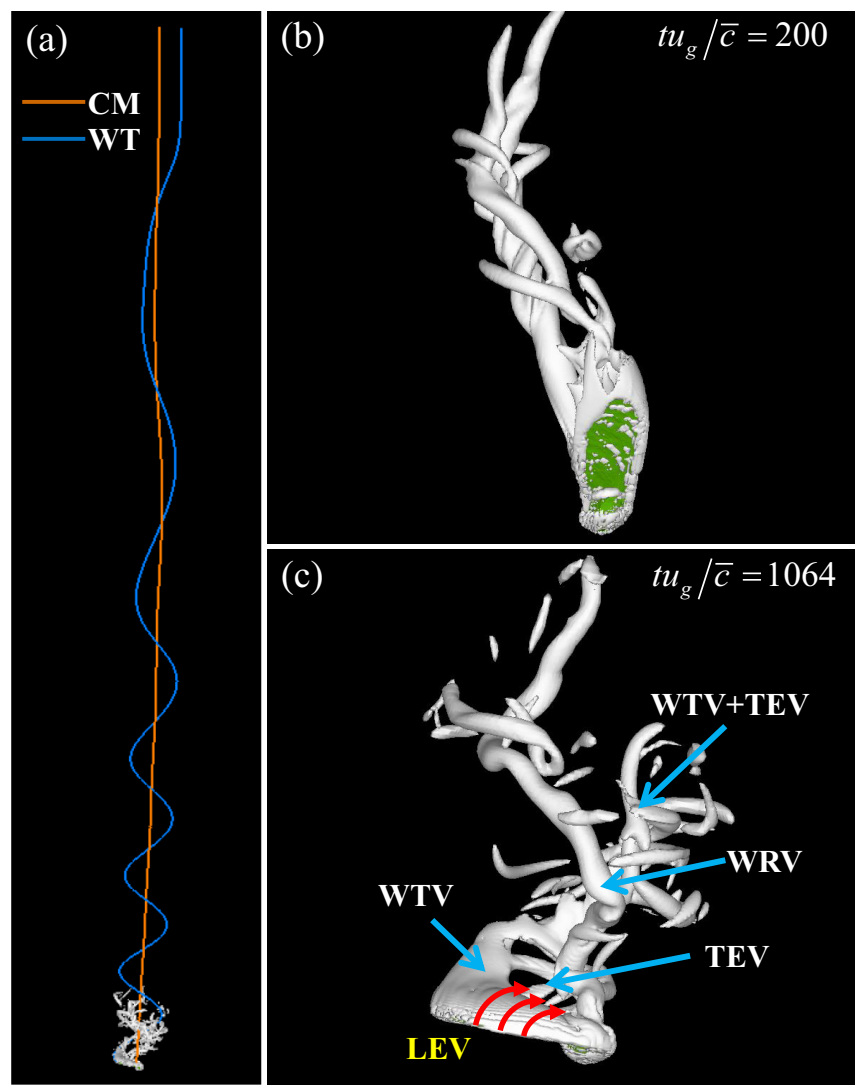

FIG. 2. (a) Trajectories of the CM and WT of the seed model during $t u_{g} / \bar{c}=0-400$; instantaneous threedimensional vortical structures around the seed at (b) $t u_{g} / \bar{c}=200$ (before periodic autorotation) and (c) $t u_{g} / \bar{c}=1064$ (during periodic autorotation). Here, the vortical structures are identified by the isosurfaces of $\lambda_{2}=-0.1$ [5]. DOI: https://doi.org/10.1103/APS.DFD.2016.GFM.V0046 


\section{FLIGHT OF A FALLING MAPLE SEED}

This work was supported by the KETEP through the Ministry of Trade, Industry and Energy (Grant No. 20152020105600), and by the NRF through the Ministry of Science and ICT of the Republic of Korea (Grant No. 2016R1E1A1A02921549).

[1] D. Lentink, W. B. Dickson, J. L. van Leeuwen, and M. H. Dickinson, Leading-edge vortices elevate lift of autorotating plant seeds, Science 324, 1438 (2009).

[2] K. Varshney, S. Chang, and Z. J. Wang, The kinematics of falling maple seeds and the initial transition to a helical motion, Nonlinearity 25, C1 (2012).

[3] D. Kim and H. Choi, Immersed boundary method for flow around an arbitrarily moving body, J. Comput. Phys. 212, 662 (2006).

[4] I. Lee, Numerical study of a freely-falling maple seed, Ph.D. thesis, Seoul National University, 2016.

[5] J. Jeong and F. Hussain, On the identification of a vortex, J. Fluid Mech. 285, 69 (1995). 